\title{
Condicionantes de género y embarazo no planificado, en adolescentes y mujeres jóvenes
}

\author{
Teresa Yago-Simón ${ }^{1 *}$ y Concepción Tomás-Aznar² \\ 1 Centro Municipal de Promoción de la Salud, Zaragoza (España). \\ 2 Facultad de Ciencias de la Salud, Universidad de Zaragoza, Zaragoza (España).
}

\begin{abstract}
Resumen: Antecedentes: Los estudios sobre comportamientos sexoreproductivos de riesgo de la población juvenil aprecian diferencias entre sexos y recomiendan incluir la perspectiva de género. El objetivo de este estudio es conocer cómo influyen los condicionantes de género en las jóvenes y si hay relación con el embarazo no planificado.

Método: Participantes: 395 mujeres entre 14 y 24 años, atendidas en consulta de anticoncepción. Instrumento: Aplicación del cuestionario sobre condicionantes de género en anticoncepción (COGANT). Análisis: Relación entre puntuaciones en el cuestionario, variables sociodemográficas y embarazo no planificado.

Resultados: Los condicionantes de género actúan más en adolescentes, extranjeras, gitanas y con menor nivel de estudios. Puntuar más elevado en el cuestionario y en los factores "Relación vincular no igualitaria" y "Rol de género tradicional", está relacionado con el embarazo no planificado.

Conclusiones: Es necesario avanzar en la igualdad y cuestionar los roles tradicionales para disminuir los embarazos no planificados en las chicas jóvenes.

Palabras clave: Anticoncepción; género; embarazo no planificado; adolescentes; jóvenes.
\end{abstract}

\section{Introducción}

Aplicar la perspectiva de género en la investigación en salud ha supuesto la apertura de nuevas hipótesis, la revisión de las metodologías y la búsqueda de resultados científicos que orienten el cambio social (Hammarstrom, 2001; Schiebinger, Klinge, Sánchez de Madariaga y Schraudner, 2011). Visibiliza las diferencias entre hombres y mujeres e indaga sobre cómo opera la red de relaciones sociales de poder que construye las posiciones de género (Serret, 2008).

Las diferencias de salud entre hombres y mujeres existen y son significativas dentro de cualquier grupo socioeconómico. Para comprender estas diferencias hay que analizar las formas complejas en que interactúan los factores biológicos y sociales. Modificar las desigualdades estructurales de género, que son las que sustentan los roles y comportamientos, tiene consecuencias en el campo de la salud (Ruiz-Cantero et al., 2007; Sen, George y Östin, 2005).

Desde hace más de veinte años, se han estudiado las actitudes, conocimientos y comportamientos sobre sexualidad y anticoncepción de la población juvenil, diferenciados por sexo (Caballero, Toro, Sánchez, y Carrera, 2009; Lucke, 1998; Sueiro, Dieguez, y Gonzalez, 1998) y se recomienda que los programas educativos, preventivos y clínicos tengan en cuenta estas diferencias (Failde, Lameiras y Bimbela, 2008;

* Dirección para correspondencia [Correspondence address]

María C. Fuentes. Departamento de Metodología de las Ciencias del Comportamiento. Facultad de Psicología. Universidad de Valencia. Av. Blasco Ibáñez, 21. 46010 Valencia (España).

E-mail: m.castillo.fuentes@uv.es
Title: Gender conditionings and unplanned pregnancies in adolescents and young girls.

Abstract: Studies about risk sexual-reproductive behaviour in young population show differences between sexes and recommend to include gender perspective. The aim of this study is to know how gender conditionings influence young women and whether there is any relationship with unplanned pregnancy.

Method: Participants: 395 women between 14 and 24 years old, attending the contraception consultation. Tool: application of the questionnaire about gender conditionings in contraception (COGANT). Analysis: relationship between scores in the question-naire, socio-demographic variables and unplanned pregnancy.

Results: Gender conditionings act more in teenage, foreign and gipsy young women and with a lower level of studies. A higher score in the questionnaire and in both factors, "Inegalitarian relationship" and "Traditional gender role", is related with an unplanned pregnancy.

Conclusions: It is necessary to make progress in equality and to question traditional roles to decrease unplanned pregnancies in young girls.

Key words: contraception; gender; unplanned pregnancy; teenagers; youth.

García-Vega, Robledo, Fernández y Fernández, 2010; López-Amorós, Schiaffino, Moncada y Pérez, 2010).

Se ha profundizado en la influencia que tienen en el comportamiento sexo- reproductivo de chicos y chicas, la cultura de origen (Puente et al., 2011), los estereotipos (Moore, 2006), la familia y el nivel de estudios (Manlove, Terry-Humen, Ikramullah y Moore, 2006), las amistades (Potard, Courtois y Rusch, 2008), la personalidad (Atkins, 2008), el sexismo y la persistencia de roles de género tradicionales y mitos románticos sobre el amor (Caricote, 2006, Larrañaga, Yubero y Yubero, 2012; Martínez, Bonilla, Gómez y Bayot, 2008).

La investigación, fundamentalmente en relación con la transmisión del SIDA, se ha centrado en conocer los comportamientos sexuales de riesgo (Ballester, Gil-Llario, RuizPalomino y Giménez-García, 2013; Bjelica y Trninic-Pjevic, 2008; Kerrigan et al., 2007) y se utilizan diversos modelos explicativos. El método Precede (Bimbela, Jiménez, Alfaro, Gutiérrez y March, 2002; Lameiras, Faílde, Bimbela y Alfaro, 2008), las teorías de las emociones anticipadas (Caballero, Toro, Sáncez y Carrera, 2009), de la autoeficacia percibida (Gómez-Zapiain, Ibaceta, Muñoz y Pardo, 1996), de la acción razonada de Ajzen y Fishbein, autoeficiencia de Bandura, modelo de salud biológica de Bayés y Ribes, analizados por López (Piña López, 2004). Rogow and Haberland (2005) señalan que tanto las normas de género como la desigual relación de poder influyen de manera negativa en la salud sexual de chicas y chicos.

A pesar de ser constantes las referencias a la pertinencia de incluir la perspectiva de género en la investigación, la mayoría de los estudios realizan un análisis por sexo. 
El rol de género influye en el comportamiento sexoreproductivo de las chicas jóvenes y es necesario conocer cómo han interiorizado los mandatos de género y su influencia en el cuidado anticonceptivo. Con esta finalidad hemos investigado en los discursos de las adolescentes y jóvenes atendidas en la consulta de anticoncepción, cuáles eran los condicionantes de género que intervenían directamente en el cuidado anticonceptivo (Yago, 2011) y con esta información hemos construido y validado un cuestionario sobre condicionantes de género en anticoncepción (COGANT) (Yago y Tomás, 2013). Nuestra investigación toma el género como categoría de análisis y objetiva la intensidad de la influencia de los condicionantes de género, a través del cuestionario.

El objetivo de este estudio es analizar la influencia de los determinantes de género en la anticoncepción en las adolescentes y mujeres jóvenes, a través de la aplicación del cuestionario COGANT, y comprobar si hay relación con el cuidado inadecuado en la anticoncepción que da lugar a un embarazo no planificado.

\section{Método}

\section{Participantes}

Se han estudiado 395 mujeres, que acudieron a la Consulta Joven de Anticoncepción del Centro Municipal de Promoción de la Salud de Zaragoza (CMPS) durante el año 2012. Su edad era entre 14 y 24 años, con una $M$ de 19.81 años y DT 2.59. Presentaban diversidad cultural y social. El $68.4 \%$ nacieron en España y el resto procedían de 24 países diferentes. Estaban estudiando $67.1 \%$ y un $4.6 \%$ eran de etnia gitana (véase Tabla 1).

Tabla 1. Descripción de la población de estudio.

\begin{tabular}{lcc}
\hline & $N$ & $\%$ \\
\hline Edad & & \\
Adolescentes (14-19 años) & 186 & 47.1 \\
Jóvenes (20-24 años) & 209 & 52 \\
Lugar de nacimiento & & \\
España & 270 & 68.4 \\
Extranjero & 125 & 31.6 \\
Iberoamericanas & 89 & 71.1 \\
Europa del Este & 19 & 14.9 \\
África & 12 & 10 \\
Otros & 5 & 4 \\
Nivel de estudios & & \\
Analfabeta y estudios primarios & 19 & 4.8 \\
Educación especial (PCPI y otros) & 47 & 11.9 \\
EGB y FP medio & 145 & 36.7 \\
Bachiller y FP superior & 64 & 16.2 \\
Grado y postgrado & 119 & 30.1 \\
No consta & 1 & 0.3 \\
Etnia & & \\
Gitana & 18 & 4.6 \\
No gitana & 377 & 95.4 \\
\hline Total & 395 & 100 \\
\hline
\end{tabular}

\section{Instrumento}

Para medir el grado de influencia de los condicionantes de género en el comportamiento anticonceptivo se ha utilizado el COGANT, de 36 ítems, diseñado y validado para esta población (Yago y Tomás 2013) (Anexo). Consta de cuatro dimensiones, con 12 factores (véase Tabla 2).

Tabla 2. Dimensiones y factores del Cuestionario sobre Condicionantes de Género en Anticoncepción (COGANT).

\begin{tabular}{ll}
\hline Dimensión & Factor \\
\hline 1. Relacional & 1.1. Actitud sumisa \\
& 1.2. Actitud ciega \\
& 1.3. Actitud pasiva \\
& 1.4. Actitud de dominio \\
2. Sobre la Maternidad & 2.1 Eje central de la identidad \\
& 2.2. No idealizada \\
3. Rol de género & 3.1. Tradicional \\
& 3.2. Inseguro \\
& 3.3. Sexual \\
4. El cuidado & 4.1. Desconfianza en los chicos \\
& 4.3. Carencia
\end{tabular}

Se recogieron datos sociodemográficos: edad, lugar de nacimiento, etnia, nivel de estudios y si habían tenido algún embarazo no planificado.

\section{Procedimiento}

El estudio se ha llevado a cabo durante el año 2012, en el CMPS, que atiende a mujeres jóvenes residentes en Zaragoza. Centro público de acceso libre, lo que garantiza que no haya sesgos de selección. Se invitó a todas las jóvenes a realizar el cuestionario en la tercera consulta, a no ser que fuera clínicamente inadecuado, no conocieran el idioma, vinieran acompañadas a la consulta, fueran analfabetas o con discapacidad psíquica.

El cuestionario fue autoadministrado y se cumplimentó en la consulta, tras la firma del consentimiento informado.

\section{Análisis estadístico}

Análisis descriptivo de los resultados obtenidos en la aplicación del cuestionario COGANT. Se analizó la $M, D T$, rango y mediana de la puntuación total obtenida.

A partir de los doce factores del cuestionario, se realizó un análisis factorial común (ejes principales) (IPA) de segundo orden con la finalidad de resumir la información. Se comprobaron las condiciones de aplicación del análisis factorial. El test de Kaiser-Meyer-Olkin (KMO) se considera inaceptable si es $<.60$, mediocre entre .60 y .70 , normal entre .70 y .80 , bueno entre .80 y .90 y excelente $>.90$. En la prueba de esfericidad de Bartlett debe rechazarse hipótesis nula de ausencia de correlación entre variables y los coeficientes de adecuación muestral deben ser superiores a .60. Para la selección de factores se utilizó el procedimiento heurístico scree-plot de Cattell, observando en el gráfico de se- 
dimentación el punto de inflexión de la línea descendente. Se aplicó rotación Varimax. Se calcularon las puntuaciones factoriales, valor mínimo 1 y valor máximo 3, y se aplicó el Test de normalidad de Kolmogorov-Smirnov; si $p<.05$ se rechaza hipótesis nula y se acepta que la distribución no es normal. Se analizó $M$ y DT de las puntuaciones factoriales. La puntuación mínima indica ausencia de influencia de los condicionantes de género y se corresponde con una actitud igualitaria y autónoma. La puntuación máxima indica que los condicionantes de género ejercen una fuerte influencia en la joven y se corresponden con una actitud discriminatoria y de pérdida de autonomía.

Se estudió la relación entre la puntuación obtenida en el cuestionario y las variables sociodemográficas. Se aplicaron las pruebas no paramétricas, U de Mann-Whitney y KruskalWallis.

Se estudió la relación entre las puntuaciones obtenidas en el cuestionario y el embarazo no planificado. Se aplicó la prueba no paramétrica, U de Mann-Whitney.

Se utilizó software SPSS 15.0.

\section{Resultados}

Durante el año 2012 acudieron a la Consulta Joven de Anticoncepción del CMPS 545 mujeres. El estudio se ha realizado con 395, ya que $74(13.94 \%)$ no reunían las condiciones de inclusión, $72(13.21 \%)$ no completaron el número de visitas necesarias y cuatro no quisieron realizarlo $(.73 \%)$.

\section{Condicionantes de género en anticoncepción y va- riables sociodemográficas}

La puntuación media obtenida en el cuestionario COGANT fue 52.95, DT 11.54, rango 36 - 94. No tiene distribución normal (test de Kolmogorov, p $<.001$ ), la mediana se sitúa en la puntuación 52. Estos valores indican el grado de influencia de los condicionantes de género en el ámbito de la contracepción (Figura 1).

La influencia de los. condicionantes está relacionada con la edad, es mayor la influencia en las adolescentes; con el lugar de nacimiento y con la etnia, es mayor la influencia en las chicas no nacidas en España y en las chicas gitanas; y con el nivel de estudios, a mayor nivel de estudios menor influencia de los condicionantes de género en anticoncepción (véase Tabla 3), diferencia que se mantiene teniendo en cuenta el grupo de edad, prueba de Kruskal-Wallis (14-19 años, $U=$ $42 Z=-3.116 p<.001,20-24$ años $U=83 Z=-3.972 p<$ $.000)$.

Se realizó un análisis factorial común (ejes principales) de segundo orden, con las puntuaciones de los doce factores del cuestionario. Medida de adecuación muestral, $K M O=$ .868 , prueba de esfericidad de Bartlett significativa, $p<.000$.
Los coeficientes de adecuación muestral de la matriz antiimagen son mayores de .60, a excepción de una puntuación que está en .41 , que corresponde al factor cuidado mutuo y se eliminó. Para la obtención de factores se utilizó el procedimiento scree-plot de Cattell, el gráfico de sedimentación se presenta en la Figura 2 y tras realizar rotación Quatrimax y Varimax, se optó por la solución aportada por la rotación Varimax. Se muestra la estructura subyacente de tres factores, con un porcentaje acumulado de varianza explicada del $51,580 \%$ (33,219\% el Factor 1, 9,426\% el Factor 2 y 8,931\% el Factor 3). (Tabla 4).

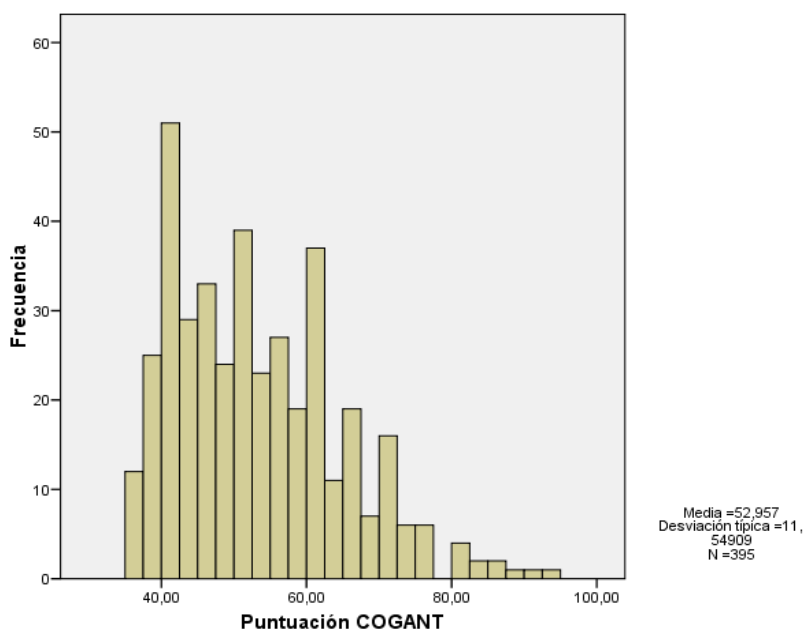

Figura 1. Puntuación obtenida en el cuestionario CONGANT.

Tabla 3. Cuestionario COGANT y variables sociodemográficas.

\begin{tabular}{|c|c|c|c|}
\hline & \multicolumn{3}{|c|}{$\begin{array}{c}\text { Puntuación en cuestionario } \\
\text { COGANT }\end{array}$} \\
\hline & $M$ & $D T$ & $p$ \\
\hline \multicolumn{4}{|l|}{ Edad } \\
\hline Adolescentes (14-19 años) & 55.57 & 11.64 & \multirow{3}{*}{$<.01 *$} \\
\hline Jóvenes (20-24 años) & 50.63 & 10.98 & \\
\hline \multicolumn{3}{|l|}{ Lugar de nacimiento } & \\
\hline España & 49.99 & 10.96 & \multirow{3}{*}{$<.01 *$} \\
\hline Extranjero & 59.37 & 10.14 & \\
\hline \multicolumn{3}{|l|}{ Etnia } & \\
\hline Gitana & 58.39 & 10.63 & \multirow{2}{*}{$<.05^{*}$} \\
\hline No gitana & 52.69 & 11.54 & \\
\hline \multicolumn{4}{|l|}{ Nivel de estudios } \\
\hline Analfabeta y estudios primarios & 61.84 & 13.84 & \multirow{5}{*}{$<.01^{* *}$} \\
\hline Educación especial (PCPI y otros) & 59.93 & 11.46 & \\
\hline EGB y FP medio & 56.71 & 10.67 & \\
\hline Bachiller y FP superior & 51.03 & 10.03 & \\
\hline Grado y postgrado & 45. 21 & 7.76 & \\
\hline
\end{tabular}




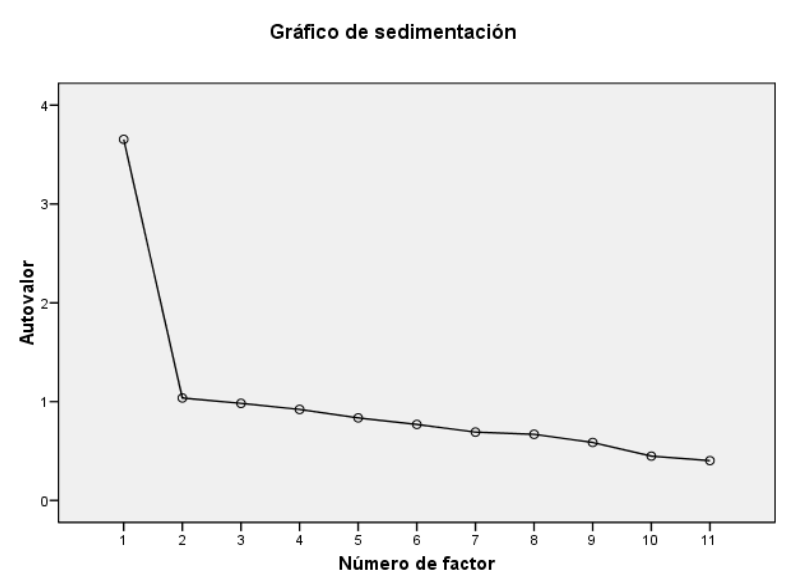

Figura 2. Gráfico de sedimentación.

El factor 1, que denominamos "Relación vincular no igualitaria", agrupa los factores que describen modalidades de relación de pareja: actitud dominante del chico y actitud sumisa, pasiva y ciega de la chica. $M, 1.472 ; D T, .365$.

El factor 2, que denominamos "Rol de género tradicional" agrupa los factores que hacen referencia a la maternidad y a los roles de género. $M, 1.529 ; D T, .371$.

El factor 3, está configurado únicamente por el Factor desconfianza en los chicos. $M, 1,490 ; D T, .856$.
Las puntuaciones factoriales de estos tres factores no tienen una distribución normal (prueba de KolmogorovSmirnov, $p<.05$ ).

Las puntuaciones factoriales de los factores "Relación vincular no igualitaria" y "Desconfianza", están relacionadas significativamente con las variables sociodemográficas, a excepción de la etnia. Las medias de las puntuaciones factoriales del factor "Rol de género tradicional" son más elevadas, indicando que la influencia de los condicionantes de género es más intensa, en adolescentes, extranjeras, gitanas y cuanto menor es el nivel de estudios (Tabla 5).

Tabla 4. Análisis factorial común. Rotación Varimax. Matriz de correlaciones.

\begin{tabular}{lccc}
\hline & \multicolumn{3}{c}{ Componente } \\
\cline { 2 - 4 } & 1 & 2 & 3 \\
\hline Actitud dominante del chico & .693 & & \\
Actitud sumisa & .679 & .328 & \\
Actitud pasiva / dejarse llevar & .640 & & \\
Rol de género inseguro & .424 & .456 & \\
Actitud ciega & .376 & & \\
Rol sexual & & .382 & \\
Maternidad eje central & & .406 & \\
Carencia cuidados maternos & & .448 & \\
Rol de género tradicional & & .444 & \\
Maternidad no idealizada & & & .756 \\
Desconfianza en los chicos & & & \\
\hline
\end{tabular}

Tabla 5. Factores y variables sociodemográficas.

\begin{tabular}{|c|c|c|c|c|c|c|c|c|c|}
\hline & \multicolumn{3}{|c|}{ Relación vincular no igualitaria } & \multicolumn{3}{|c|}{ Rol de género tradicional } & \multicolumn{3}{|c|}{ Desconfianza } \\
\hline & $M$ & $D T$ & $p$ & $M$ & $D T$ & $p$ & $M$ & $D T$ & $p$ \\
\hline \multicolumn{10}{|l|}{ Edad } \\
\hline Adolescentes & 1.55 & .37 & \multirow[t]{2}{*}{$.000^{*}$} & 1.61 & .37 & \multirow[t]{2}{*}{$.000^{*}$} & 1.55 & .89 & \multirow[t]{2}{*}{$.000^{*}$} \\
\hline Jóvenes & 1.40 & .34 & & 1.46 & .36 & & 1.44 & .83 & \\
\hline \multicolumn{10}{|l|}{ Lugar de nacimiento } \\
\hline España & 1.40 & .36 & \multirow[t]{2}{*}{$.000^{*}$} & 1.42 & .32 & \multirow[t]{2}{*}{$.000^{*}$} & 1.42 & .80 & \multirow[t]{2}{*}{$.010^{*}$} \\
\hline Extranjero & 1.63 & .32 & & 1.78 & .36 & & 1.66 & .93 & \\
\hline \multicolumn{10}{|l|}{ Etnia } \\
\hline Gitana & 1.57 & .33 & \multirow[t]{2}{*}{$.117^{*}$} & 1.75 & .30 & \multirow[t]{2}{*}{$.004^{*}$} & 1.50 & .86 & \multirow[t]{2}{*}{$.889 *$} \\
\hline No gitana & 1.47 & .37 & & 1.52 & .37 & & 1.49 & .86 & \\
\hline \multicolumn{10}{|l|}{ Nivel de estudios } \\
\hline Analfabeta/ primarios & 1.69 & .45 & \multirow{5}{*}{$.000 * *$} & 1.82 & .36 & \multirow{5}{*}{$.000^{* *}$} & 1.63 & .96 & \multirow{5}{*}{$.000^{* *}$} \\
\hline Educación especial... & 1.65 & .37 & & 1.73 & .38 & & 2.04 & .99 & \\
\hline EGB y FP medio & 1.58 & .37 & & 1.64 & .34 & & 1.60 & .90 & \\
\hline Bachiller y FP Sup. & 1.42 & .32 & & 1.48 & .33 & & 1.45 & .83 & \\
\hline Grado y postgrado & 1.27 & .25 & & 1.30 & .30 & & 1.15 & .53 & \\
\hline
\end{tabular}

* U de Mann-Whitney

** Kruskal-Wallis

\section{Condicionantes de género en anticoncepción y em- barazo no planificado}

De las 395 jóvenes del estudio, 99 (25.1\%) han tenido, al menos, un embarazo no planificado y está relacionado significativamente con la puntuación obtenida en el cuestionario COGANT (véase Tabla 6) y con las puntuaciones de los factores Relación vincular no igualitaria, Rol de género tradi- cional y Desconfianza, en el sentido de que la influencia de los condicionantes de género en anticoncepción es más intensa en las jóvenes que han tenido un embarazo no planificado. 
Tabla 6. Cuestionario COGANT y embarazo no planificado.

\begin{tabular}{lccc}
\hline & \multicolumn{3}{c}{ Puntuación en cuestionario COGANT } \\
\cline { 2 - 5 } & $M$ & $D T$ & $p$ \\
\hline Embarazo no planificado & \multicolumn{2}{c}{$N=99$} & \\
SI & 59.44 & 11.1 & $<.01 *$ \\
NO & $N=296$ & & \\
& 50.79 & 10.88 & \\
\hline
\end{tabular}

*U de Mann-Whitney

Teniendo en cuenta las variables sociodemográficas, se mantiene la significación por grupo de edad, (14-19 años $U$ $=2325, Z=-3.075, p<.002,20-24$ años, $U=1784, Z=$ $5.987, p<.000$ ), por etnia, (no gitana $U=6631, Z=-7.056$ , $p<.000$, gitana $U=16.5, Z=-2.101, p<.034)$ y por estudios, test de Kruskal-Wallis, significación aportada por el segmento EGB-FP medio (Chi $=6.998, p<.01$ ). No se mantiene la significación descartando la influencia del lugar de origen.

Hay relación significativa entre embarazo no planificado y las puntuaciones factoriales. "Relación vincular no igualitaria", $U=9109.0, Z=-5.641, p<.000$. "Rol de género tradicional" $U=8731.0, Z=-6.023, p<.000$. "Desconfianza", $U$ $=11602.0, Z=-.4 .110, p<.000$.

\section{Conclusiones}

Aplicar la perspectiva de género en la investigación en anticoncepción, nos ha aportado información sobre cómo influyen los condicionantes de género en las adolescentes y jóvenes y sobre la relación existente entre condicionantes y embarazo no planificado.

Los condicionantes de género en el ámbito de la contracepción, que sitúan a las chicas en una posición de desigualdad en las relaciones afectivo-sexuales y que atribuyen a la maternidad un papel central en la configuración de la identidad femenina, no tienen una gran influencia en las chicas jóvenes.

El cuestionario está focalizado en los ámbitos relacionados con el comportamiento sexo-reproductivo y no hay estudios que tengan este mismo objetivo. No obstante, referencias similares encontramos en los estudios sobre sexismo (Díaz-Aguado y Carvajal, 2011; Ferragut, Blanca y OrtizTallo, 2013), sobre estereotipos sexuales (Ruiz, Ballester, Gil,

\section{Referencias}

Afable-Munsuz, A. y Brindis, C. D. (2006). Acculturation and the sexual and reproductive health of latino youth in the united states: A literature review. Perspectives on Sexual and Reproductive Health, 38(4), 208-219. DOI:10.1363/3820806

Atkins, R. (2008). The association of childhood personality on sexual risk taking during adolescence. Journal of School Health, 78(11), 594-600. DOI:10.1111/j.1746-1561.2008.00351.x

Ballester, R., Gil-Llario, M. D., Ruiz-Palomino, E. y Giménez-García, C. (2013). Autoeficacia en la prevención sexual del sida: La influencia del género. Anales de Psicología, 29(1), 76-82. DOI: 10.6018/analesps.29.1.124601
Giménez y Salmerón, 2010) y sobre los mitos románticos y las relaciones de sumisión (Larrañaga et al., 2012; Martínez et al., 2008).

Las jóvenes de este estudio presentan gran diversidad sociocultural y hemos constatado que las variables sociodemográficas, edad, lugar de origen, nivel de estudios y etnia interactúan con el género. La edad y el nivel de estudios modulan la influencia social negativa que ejercen sobre las mujeres los roles y estereotipos sexuales discriminatorios. Cabe preguntarse si el motivo de que las adolescentes estén colocadas en posiciones más desiguales que las adultas jóvenes, sin ser la posición predominante, es un cambio de tendencia social o que el proceso de maduración da lugar a mayor autonomía en las mujeres.

Hemos comprobado que el embarazo no planificado está relacionado con el género, manifestado en relaciones vinculares no igualitarias y una concepción tradicional de los roles femeninos. Hay estudios sobre relaciones no igualitarias y uso del preservativo, violencia y/o SIDA (Dunkle y Decker, 2013; Jewkes et al., 2006; Kerrigan et al., 2007; Machel, 2001). Afable-Munsuz and Brindis (2006), Saxena et al. (2006) y Vargas, Henao y González (2007) resaltan la importancia de la escolarización y el contexto sociocultural y Bjelica and Trninic-Pjevic (2008), Geldstein and Pantelides (1997) y Higgins and Hirsch (2008), la influencia del género y la clase social en la anticoncepción.

Es necesario conocer los factores que influyen en el comportamiento anticonceptivo, para poder vencer los obstáculos que impiden mejorar los índices de embarazos no planificados entre las jóvenes. La cultura, la familia, la historia personal, la educación y atención sanitaria pueden favorecer o prevenir los embarazos no planificados, y el género se configura como otro determinante que actúa negativamente en las jóvenes. Por tanto, prevenir embarazos no planificados en esta población va más allá de mejorar el nivel de educación, la información sobre sexualidad y anticoncepción y el acceso y calidad de los servicios de salud, es necesario también cuestionar los modelos tradicionales de género. Hay que trabajar por la libertad e igualdad real entre chicos y chicas, enseñarles a decodificar las relaciones vinculares con rasgos de dominación-sumisión y ampliar espacios de desarrollo personal y profesional, más allá de la maternidad como único proveedor de valor personal y social.
Bimbela, J. L., Jiménez, J. M., Alfaro, N., Gutiérrez, P. y March, J. C. (2002). Uso del profiláctico entre la juventud en sus relaciones de coito vaginal. Gaceta Sanitaria, 16(4), 298-307. Recuperado de http://gacetasanitaria.org/

Bjelica, A. y Trninic-Pjevic, A. (2008). Review of identified factors influencing contraceptive use. Medicinski Pregled, 61(3/4), 151-155. Recuperado de http://www.dlv.org.rs/izdavacka_medicinski.htm

Caballero, A., Toro, I., Sánchez, F. y Carrera, P. (2009). Las emociones y el género en la conducta sexual de riesgo en adolescentes. Revista de Psicologia Social, 24(3), 349-361. DOI:10.1174/021347409789050551 
Caricote, E. (2006). Influencia de los estereotipos de género en la salud sexual en la adolescencia. Educere, 10(34), 462-470. Recuperado de http://www.human.ula.ve/adocente/educere/

Díaz-Aguado, M. J., y Carvajal, M. I. (2011). Igualdad y prevención de la violencia de género en la adolescencia. Madrid: Ministerio de Sanidad, Política Social e Igualdad.

Dunkle, K. L., y Decker, M. R. (2013). Gender-based violence and HIV: Reviewing the evidence for links and causal pathways in the general population and high-risk groups. American Journal of Reproductive Immunology, 69, 20-26. DOI:10.1111/aji.12039

Failde, J. M., Lameiras, M., y Bimbela, J. L. (2008). Prácticas sexuales de chicos y chicas españoles de 14-24 años de edad. Gaceta Sanitaria, 22(6), 511-519. Recuperado de http://gacetasanitaria.org/

Ferragut, M., Blanca, M. J., y Ortiz-Tallo, M. (2013). Psychological values as protective factors against sexist attitudes in preadolescents. Psicothema, 25(1), 38-42. DOI:10.7334/psicothema2012.85

García-Vega, E., Robledo, E. M., Fernández, P. y Fernández, R. R. (2010). Inflluencia del sexo y del género en el comportamiento sexual de una población adolescente. Psicothema, 22(4), 606-612. Recuperado de www.psicothema.com

Geldstein, R. N., y Pantelides, E. A. (1997). Double subordination, double risk: Class, gender and sexuality in adolescent women in Argentina. Reproductive Health Matters, 9, 121-131. DOI:10.1016/S09688080(97)90015-X

Gómez-Zapiain, J., Ibaceta, P., Muñoz, F., y Pardo, E. (1996). Autoeficacia percibida en relación al comportamiento sexual y contraceptivo de los jóvenes. Revista de Psicología General y Aplicada, 49(1), 173-183.

Hammarstrom, A. (2001). Medicine has much to learn from gender studies. Lakartidningen, 98(11), 1222-1227. Recuperado de http://www.lakartidningen.se/

Higgins, J. A., y Hirsch, J. S. (2008). Pleasure, power, and inequality: Incorporating sexuality into research on contraceptive use. American Journal of Public Health, 98(10), 1803-1813. DOI:10.2105/AJPH.2007.115790

Jewkes, R., Dunkle, K., Nduna, M., Levin, J., Jama, N., Khuzwayo, N., Koss, M., Puren, A. y Duvvury, N. (2006). Factors associated with HIV sero-status in young rural south african women: Connections between intimate partner violence and HIV. International Journal of Epidemiology, 35(6), 1461-1468. DOI: 10.1093/ije/dyl218

Kerrigan, D., Andrinopoulos, K., Johnson, R., Parham, P., Thomas, T., y Ellen, J. M. (2007). Staying strong: Gender ideologies among africanamerican adolescents and the implications for HIV/STI prevention. Journal of Sex Research, 44(2), 172-180. DOI:10.1080/00224490701263785

Lameiras, M., Faílde, J. M., Bimbela, J. L. y Alfaro, O. (2008). Uso de preservativo masculino en las relaciones con coito vaginal de jóvenes españoles entre catorce y veinticuatro años. Diversitas-Perspectivas en Psicología, (2), 401-415.

Larrañaga, E., Yubero, S. y Yubero, M. (2012). Influencia del género y del sexo en las actitudes sexuales de estudiantes universitarios españoles. Summa Psicológica, 9(2), 5-13. Recuperado de http://www.summapsicologica.cl/index.php/summa

López-Amorós, M., Schiaffino, A., Moncada, A. y Pérez, G. (2010). Factores asociados al uso autodeclarado de la anticoncepción de emergencia en la población escolarizada de 14 a 18 años de edad. Gaceta Sanitaria, 24(5), 404-409. Recuperado de http://gacetasanitaria.org/

Lucke, J. C. (1998). Gender roles and sexual behavior among young women. Sex Roles, 39(3-4), 273-297. Recuperado de http://www.springer.com/psychology/personality $+\% 26+$ social + psych ology/journal/11199

Machel, J. Z. (2001). Unsafe sexual behaviour among schoolgirls in mozambique: A matter of gender and class. Reproductive Health Matters, 9(17), 82-90. DOI:10.1016/S0968-8080(01)90011-4
Manlove, J. S., Terry-Humen, E., Ikramullah, E. N., y Moore, K. A. (2006). The role of parent religiosity in teens' transitions to sex and contraception. The Journal of Adolescent Health, 39(4), 578-587. Recuperado de http://www.jahonline.org/home

Martínez, I., Bonilla, A., Gómez, L. y Bayot, A. (2008). Identidad de género y afectividad en la adolescencia: Asimetrías relacionales y violencia simbólica. Anuario de Psicología, 39(1), 109-118. Recuperado de http://www.ub.edu/psicologia/castellano/anuario-de-psicologia

Moore, A. M. (2006). Gender role beliefs at sexual debut: Qualitative evidence from two brazilian cities. International Family Planning Perspectives, 32(1), 45-51. http://www.guttmacher.org

Piña López, J. A. (2004). Eventos disposicionales que probabilizan la práctica de conductas de riesgo para el VIH/SIDA. Anales de Psicología, 20(1), 23-32. Recuperado de http://www.um.es/analesps/

Potard, C., Courtois, R. y Rusch, E. (2008). The influence of peers on risky sexual behaviour during adolescence. The European Journal of Contraception y Reproductive Health Care, 13(3), 264-270. DOI:10.1080/13625180802273530

Puente, D., Zabaleta, E., Rodriguez-Blanco, T., Cabanas, M., Monteagudo, M., Pueyo, M. J., ... Bolíbar, B (2011). Diferencias de género en conductas sexuales de riesgo en adolescentes en cataluña. Gaceta Sanitaria, 25(1), 13-19. Recuperado de http://gacetasanitaria.org/

Rogow, D.y Haberland, N. (2005). Educación sobre sexualidad y relaciones: Hacia una perspetiva de estudios sociales. Sex Education, 5(4), 333-344. Recuperado de http://www.popcouncil.org/pdfs/SE 54 esp.pdf

Ruiz, E., Ballester, R., Gil, M. D., Giménez, C. y Salmerón, P. (2010). ¿Cambian los estereotipos de género a lo largo de la adolescencia? Revista INFAD de Psicología, 1(2), 321-329. Recuperado de http://infad.eu/RevistaINFAD/

Ruiz-Cantero, M. T., Vives-Cases, C., Artazcoz, L., Delgado, A., Garcia Calvente, M. M., Miqueo, C., ... Valls C. (2007). A framework to analyse gender bias in epidemiological research. Journal of Epidemiology and Community Health, 61, 46-53. DOI:10.1136/jech.2007.062034

Saxena, S., Copas, A. J., Mercer, C., Johnson, A. M., Fenton, K., Erens, B., ... Wellings, K. (2006). Ethnic variations in sexual activity and contraceptive use. Contraception, 74(3), 224-233. DOI:10.1016/j.contraception.2006.03.025.

Schiebinger, L., Klinge, I., Sánchez de Madariaga, I. y Schraudner, M., eds. (2011-2013). Gendered Innovations in Science, Health y Medicine, Engineering, and Environment. Recuperado de http://genderedinnovations.stanford.edu/

Sen, G., George, A. y Östin, P. (2005). Engendering international health:The challange of equity. Working Papers for Population and Development Studies, Harvard School of Public Health, 12(2).

Serret, E. (2008). Qué es y para qué es la perspectiva de género. Méjico: Instituto de la Mujer Oaxaqueña.

Sueiro, E., Dieguez, J. L., y Gonzalez, A. (1998). Jóvenes que realizan estudios universitarios: salud sexual y reproductiva. Atencion Primaria, 21(5), 283-288. Recuperado de http://zl.elsevier.es/es/revista/atencionprimaria- 27

Vargas, E., Henao, J. y González, C. (2007). Toma de decisiones sexuales y reproductivas en la adolescencia. Acta Colombiana de Psicología, 10, 10491063. Recuperado http://regweb.ucatolica.edu.co/publicaciones/psicologia/ACTA/

Yago, T., y Tomás, C. (2013). Condicionantes de género en anticoncepción: Diseño y validación de un cuestionario. Atención Primaria, 45 (8), 418-425

Yago, T. (2011). Jóvenes, anticoncepción y género. Zaragoza: Seminario Interdisciplinar de Estudios de la Mujer. Universidad de Zaragoza.

(Artículo recibido: 13-11-2013; revisado: 15-09-2014; aceptado: 09-01-2015) 
Anexo: Cuestionario COGANT, dimensiones, factores e ítems. (Yago y Tomás, 2013)

Dimensión relacional

Actitud Sumisa

- $\quad$ Creo que estoy embarazada, pero no se lo he dicho a él por no preocuparle.

- Cuando mi chico no tiene mucho deseo sexual, sospecho que está interesado por otra chica.

- $\quad$ Si lo pierdo no tengo nada, no soy nada.

- Me dio pena y tuve relaciones con él, aunque yo no quería.

- $\quad$ Cuando me pide perdón, me da tanta pena que vuelvo. Actitud ciega

- $\quad$ Con éste va a ser diferente, llevamos dos meses pero es pareja estable.

- Cuando estoy enamorada no pienso en cuidarme en las relaciones sexuales.

- Sabía que a otras chicas les había "puesto los cuernos", pero estaba segura que conmigo sería diferente. Actitud pasival dejarse llevar

- $\quad$ Cuando bebo mucho, me comporto muy diferente en lo que se refiere al sexo.

- Me siento tan apoyada por él, como él por mí; creo que los dos ponemos igual en esta relación.

- Me digo muchas veces, no voy a hacerlo por hacerlo, pero... al final lo vuelvo a hacer.

- $\quad$ Creo que soy muy importante para él.

- $\quad$ Desde que estamos juntos, he dejado de ir con mis amigas. Actitud de dominio del chico

- $\quad$ Si insisto en el uso de preservativo se pone como loco, grita, fuera de sí, y lo dejo porque sino es peor.

- $\quad$ El no se pone el preservativo y, por no discutir, no hablo.

- Cuando mantengo relaciones sexuales tengo la impresión de que él no cuida demasiado de mí.

- $\quad$ Temo que si le digo que no me gusta lo que quiere que hagamos, me deje.

- $\quad$ Cuando son novios se ponen celosos y ya no te dejan hacer nada.

Dimensión: Maternidad

\section{Eje central de la identidad}

- $\quad$ No me parece que por ser joven no se pueda ser madre, me creo capaz de hacerlo.

- Si tuviera que elegir entre ser madre y seguir con mis proyectos (estudios, viajes, trabajo,...) elegiría la maternidad.

- $\quad$ Mi vida no tendría sentido si no puedo ser madre, en un futuro.

No idealizada

- $\quad$ Cuando pienso en mi madre creo que tampoco es tan maravillosa la maternidad.

- $\quad$ Entre mi gente es normal ser madre siendo joven.

Dimensión: Rol de género

\section{Rol Tradicional}

- $\quad$ Si alguien ha de cuidar en las relaciones sexuales, es la chica.

- Todas las mujeres desean ser madres.

- $\quad$ Se es mujer, cuando se es madre.

Rol Inseguro

- $\quad$ Siente muchísima vergüenza cuando tengo que pedir la píldora del día siguiente.

- Me he preguntado muchas veces, ¿seré estéril?

- A veces he pensado: no voy a hacerlo con él a ver si así me valora y me quiere más.

- Ya no eres la misma cuando has decidido abortar, es como si fueras una mala madre, aunque no tenga porqué ser así.

- $\quad$ Si se rompe la pareja es como si yo no hubiera sido capaz de mantenerla y estar bien los dos.

Rol sexual

- $\quad$ Me da vergüenza que me vean que llevo preservativos.

- $\quad$ No tomo la iniciativa nunca, prefiero dejarme llevar por él.

Dimensión: El cuidado

Confianza: No confío en que ningún chico se preocupe y cuide de mí

Carencia básica: Cuando veo a madres que quieren y cuidan a sus hijos, pienso que porqué yo he tenido tan mala suerte. 